\title{
Sequential Design Strategies for Mean Response Surface Metamodeling via Stochastic Kriging with Adaptive Exploration and Exploitation
}

\author{
X. Chen ${ }^{\mathrm{a}, *}$, Q. Zhou ${ }^{\mathrm{b}}$ \\ ${ }^{a}$ Grado Department of Industrial and Systems Engineering, Virginia Tech, Blacksburg, VA 24601, \\ USA \\ ${ }^{b}$ Department of Systems and Industrial Engineering, University of Arizona, Tucson, AZ 85721, \\ USA
}

\begin{abstract}
Stochastic kriging (SK) methodology has been known as an effective metamodeling tool for approximating a mean response surface implied by a stochastic simulation. In this paper we provide some theoretical results on the predictive performance of SK, in light of which novel integrated mean squared error-based sequential design strategies are proposed to apply SK for mean response surface metamodeling with a fixed simulation budget. Through numerical examples of different features, we show that SK with the proposed strategies applied holds great promise for achieving high predictive accuracy by striking a good balance between exploration and exploitation.
\end{abstract}

Keywords:

Simulation, Sequential experimental design, Simulation metamodeling, Simulation analysis and methodology

\section{Introduction}

Complex computer simulation models of proposed or existing real systems are often used to aid system design. This usually happens when it is impractical to construct multiple prototype versions of the real system, or other constraints such as cost or time prohibit experimentation with the real system. Analysts often use

\footnotetext{
*Corresponding author. Tel.: +1 540 2314695; fax: +1 5402313322.

Email addresses: xchen6@vt .edu (X. Chen), zhouq@email.arizona.edu (Q. Zhou)
} 
the simulation model as a surrogate to do the system design. However, simulation models themselves can be quite complicated, and the decision to build and use a simulation model of a large-scale, complex system represents a nontrivial investment of time and money. Furthermore, in applications where intense simulation is necessary to evaluate even one scenario or where it is required to learn the impact of many "what if" scenarios, simulation cannot deliver the desired answer in a timely manner. As a result, a metamodel is often built on outputs from simulations run at some selected design points to "map" the performance response surface as a function of the controllable decision variables, or uncontrollable environmental variables, to approximate the behavior of the original simulation model. This metamodel can be used as an accurate, drop-in replacement for the simulation model as if the simulation can be run "on demand." Successful applications have been recorded in many cases, including urban transportation analysis (Osorio and Bierlaire, 2013), polymerization reaction process study (Ouyang et al., 2017) and analysis of manufacturing process with switching output regimes (Santos and Santos, 2016).

The kriging methodology has been very popular in various engineering disciplines for approximating the output of deterministic computer experiments (i.e., the same output is produced if the simulation is run twice at the same design point); see, for instance, Santner et al. (2003). To build a high-quality metamodel such as kriging with a given simulation budget to expend, a carefully designed simulation experiment is required. The literature on experimental designs for deterministic computer experiments abounds and various non-sequential design schemes have been proposed, for instance, Latin hypercube designs (LHDs) (McKay et al., 1979), orthogonal array based LHDs (Tang, 1993), uniform designs (Fang et al., 2000), and maximum entropy designs (Shewry and Wynn, 1987); see more details from Section 5.5 of Kleijnen (2015). More recently, Gauthier and Pronzato $(2014,2016)$ investigate spectral approximations of the integrated mean squared error (IMSE) and the truncated-IMSE and obtain efficient approximations of IMSE-optimal quadrature designs. Compared to the aforementioned non-sequential designs that choose all design points up front, sequential designs can offer a huge advantage in that they improve budget allocation efficiency by learning information from previous experiment runs and allocating the remaining simulation budget more wisely.

Sequential design strategies for global metamodeling have been studied in the context of deterministic computer experiments, to perform either prediction or optimization (Kleijnen, 2017). With respect to metamodeling for prediction, the interest lies in constructing sequential designs for a metamodel to achieve high 
predictive accuracy across the entire design space. For example, Ranjan et al. (2008) propose a sequential design method for estimating a contour (also called a level set of iso-surface) of a computer code. In reliability engineering, Bect et al. (2012) estimate the probability of failure with a sequential strategy using stepwise uncertainty reduction. Kleijnen and van Beers (2004) propose an applicationdriven sequential strategy using cross-validation and jackknifing to estimate the variances of computer outputs for candidate design points. Their approach tends to add more design points to those design regions with interesting input/output behavior. As to metamodeling for optimization, the focus is on establishing sequential methods to seek the global optimum. The pioneering work that introduces the efficient global optimization (EGO) approach (Jones et al., 1998) has inspired the development of many EGO variants for solving various types of optimization problems; the interested reader is referred to details from Section 6.2 of Kleijnen (2017) and Section 6.3 of Kleijnen (2015) and references therein.

In recent years, kriging related research for stochastic simulation (or random computer experiments) has flourished, leading to a plethora of theoretical and empirical work on a wide range of topics. A majority of these studies have been dedicated to efficiently metamodeling the mean response surface implied by a stochastic simulation, e.g., van Beers and Kleijnen (2008), Ankenman et al. (2010), Ng and Yin (2012) and Mehdad and Kleijnen (2015). There also exist research studies that either focus on jointly metamodeling the underlying mean and simulation variance response surfaces (e.g., Kersting et al., 2007; Boukouvalas and Cornford, 2009; Robinson et al., 2010; Marrel et al., 2012; Wang and Chen, 2016; Binois et al., 2016) or concern about quantile-based response surface approximation (e.g., Yang et al., 2008; Chen and Kim, 2013; Bekki et al., 2014; Picheny et al., 2013; Chen and Kim, 2016), to better cope with heteroscedasticity present in the stochastic simulation outputs.

The stochastic kriging (SK) methodology proposed by Ankenman et al. (2010) distinguishes itself as an effective metamodeling tool for approximating a mean response surface by correctly accounting for both sampling uncertainty inherent in a stochastic simulation and the response-surface uncertainty. Recently, fruitful results have been achieved both with respect to in-depth theoretical investigations and regarding successful applications of SK (e.g., Liu and Staum, 2010; Chen et al., 2012, 2013; Chen and Kim, 2014, 2016).

With respect to metamodeling for mean response prediction in the stochastic simulation setting, some in-depth work has been conducted regarding asymptotic properties of kriging prediction with nugget and the implications on nonsequential experimental designs with a large simulation budget are provided (Gratiet 
and Garnier, 2015). However, a systematic account of sequential designs with a fixed simulation budget has yet to be established, despite the earlier efforts by van Beers and Kleijnen (2008), Ng and Yin (2012), Ajdari and Mahlooji (2014) and Mehdad and Kleijnen (2015). In particular, very little work has been done on devising efficient experimental designs for applying the SK methodology. There exist some sequential design strategies proposed by Chen and Zhou (2014), nevertheless their results are preliminary and lack a theoretical grounding.

In this paper we aim to provide a first step toward establishing efficient sequential design strategies to implement SK for mean response prediction with a fixed simulation budget. We provide some theoretical results on the predictive performance of SK, in light of which we propose novel IMSE-based sequential design strategies that show great promise in striking a good balance between exploration (namely, selecting new design points to perform simulations for reducing response-surface uncertainty) and exploitation (namely, allocating simulation budget to existing design points for reducing sampling uncertainty). The remainder of this paper is organized as follows. Section 2 provides a review on SK. Section 3 studies some properties of SK theoretically, which leads to the proposed sequential design strategies for implementing SK in Section 4 . The predictive performances of SK with different design strategies used are demonstrated through numerical examples in Section 5. Section 6 concludes the paper.

\section{A Review on Stochastic Kriging}

We provide a brief review on the stochastic kriging (SK) methodology in this subsection. SK assumes the following model to represent the simulation output obtained on the $j$ th simulation replication at design point $\mathbf{x} \in \mathscr{X} \subset \mathbb{R}^{d}$,

$$
\mathscr{Y}_{j}(\mathbf{x})=\mathrm{Y}(\mathbf{x})+\varepsilon_{j}(\mathbf{x})=\mathbf{f}(\mathbf{x})^{\top} \beta+\mathrm{M}(\mathbf{x})+\varepsilon_{j}(\mathbf{x}),
$$

where $\mathrm{Y}(\mathbf{x})$ denotes the unknown performance-measure surface of interest, and $\varepsilon_{j}(\mathbf{x})$ represents the simulation noise that has mean zero and variance $\mathrm{V}(\mathbf{x}):=$ $\operatorname{Var}\left(\varepsilon_{j}(\mathbf{x})\right)$. Furthermore, $\mathbf{f}(\mathbf{x})$ is a $p \times 1$ vector of known functions of $\mathbf{x}$ and $\beta$ is a $p \times 1$ vector of unknown parameters.

As treated in the design and analysis of deterministic computer experiments literature (Santner et al., 2003), SK postulates that $\mathrm{M}(\cdot)$ represents a second-order stationary mean-zero Gaussian random field. That is, $\mathrm{M}(\mathbf{x})$ can be regarded as being sampled randomly from a space of mappings $\mathbb{R}^{d} \rightarrow \mathbb{R}$, in which functions are assumed to exhibit spatial correlation. Specifically, there exists a spatial correlation function $\mathscr{R}(\cdot ; \theta)$ that measures the correlation of the values of 
$\mathrm{M}\left(\mathbf{x}_{i}\right)$ and $\mathrm{M}\left(\mathbf{x}_{\ell}\right)$. This correlation is determined by the distance between $\mathbf{x}_{i}$ and $\mathbf{x}_{\ell}$ measured along each of the $d$ dimensions, and the $d \times 1$ parameter vector $\theta=\left(\theta_{1}, \theta_{2}, \ldots, \theta_{d}\right)^{\top}$ controls how quickly the spatial correlation diminishes as the two points become farther apart in each direction. Commonly used correlation functions include the Gaussian correlation function, Matern correlation functions, and the exponential correlation function (see Chapter 4 of Rasmussen and Williams, 2006); we choose to use the popular Gaussian correlation function $\mathscr{R}\left(\mathbf{x}_{i}-\mathbf{x}_{\ell} ; \theta\right)=\exp \left(-\sum_{r=1}^{d} \theta_{r}\left(x_{i r}-x_{\ell r}\right)^{2}\right)$ in this paper. Given a correlation function, the implied covariance function is

$$
\operatorname{Cov}\left(\mathrm{M}\left(\mathbf{x}_{i}\right), \mathrm{M}\left(\mathbf{x}_{\ell}\right)\right)=\tau^{2} \mathscr{R}\left(\mathbf{x}_{i}-\mathbf{x}_{\ell} ; \theta\right),
$$

where $\tau^{2}$ denotes the variance of $\mathrm{M}(\mathbf{x})$ for all $\mathbf{x} \in \mathscr{X}$. We note that in contrast to the stochastic nature of $\mathrm{M}$ referred to as extrinsic uncertainty by Ankenman et al. (2010), the intrinsic uncertainty represented by $\varepsilon$ is inherent in a stochastic simulation output; we further assume that $\mathrm{M}$ and $\varepsilon$ are independent.

In the stochastic simulation setting, an experimental design consists of designpoint locations and the corresponding numbers of replications to conduct simulations, e.g., $\mathscr{D}=\left\{\left(\mathbf{x}_{i}, n_{i}\right), i=1,2, \ldots, k\right\}$. Denote the sample average simulation response at $\mathbf{x}_{i}$ by $\mathscr{Y}\left(\mathbf{x}_{i}\right)$ and let the vector of the sample average simulation responses be $\overline{\mathscr{Y}}=\left(\overline{\mathscr{Y}}\left(\mathbf{x}_{1}\right), \overline{\mathscr{Y}}\left(\mathbf{x}_{2}\right), \ldots, \overline{\mathscr{Y}}\left(\mathbf{x}_{k}\right)\right)^{\top}$. Following the aforementioned description, SK represents the sample average simulation response by

$$
\overline{\mathscr{Y}}\left(\mathbf{x}_{i}\right)=\frac{1}{n_{i}} \sum_{j=1}^{n_{i}} \mathscr{Y}_{j}\left(\mathbf{x}_{i}\right)=\mathrm{Y}\left(\mathbf{x}_{i}\right)+\bar{\varepsilon}\left(\mathbf{x}_{i}\right),
$$

where $\bar{\varepsilon}\left(\mathbf{x}_{i}\right):=n_{i}^{-1} \sum_{j=1}^{n_{i}} \varepsilon_{j}\left(\mathbf{x}_{i}\right)$ denotes the simulation error associated with the performance-measure point estimate $\overline{\mathscr{Y}}\left(\mathbf{x}_{i}\right), i=1,2, \ldots, k$. The simulation errors $\varepsilon_{1}\left(\mathbf{x}_{i}\right), \varepsilon_{2}\left(\mathbf{x}_{i}\right), \ldots$ are naturally independent and identically distributed (i.i.d.) across replications in the stochastic simulation setting. In this paper we do not consider using common random numbers ( $\mathrm{CRN}$ is a widely used variance reduction technique that tends to introduce positive correlation between simulation outputs obtained at a pair of distinct design points on the same replication, see Law, 2015). Consequently, $\operatorname{Corr}\left(\varepsilon_{j}\left(\mathbf{x}_{i}\right), \varepsilon_{j}\left(\mathbf{x}_{\ell}\right)\right)=0$ for $\mathbf{x}_{\ell} \neq \mathbf{x}_{i}$; furthermore, the variance-covariance matrix of $\bar{\varepsilon}:=\left(\bar{\varepsilon}\left(\mathbf{x}_{1}\right), \bar{\varepsilon}\left(\mathbf{x}_{2}\right), \ldots, \bar{\varepsilon}\left(\mathbf{x}_{k}\right)\right)^{\top}, \Sigma_{\varepsilon}$, reduces to a diagonal matrix.

SK considers building a linear predictor of the form $\lambda_{0}+\lambda^{\top} \overline{\mathscr{Y}}$ to predict the performance-measure $\mathrm{Y}\left(\mathbf{x}_{0}\right)$ at any point $\mathbf{x}_{0} \in \mathscr{X}$, such that the location dependent 
weights $\lambda_{0}$ and $\lambda$ are chosen to minimize the resulting mean squared error (MSE) of prediction. Assuming that the model parameters $\beta, \tau^{2}, \theta$ and $\Sigma_{\varepsilon}$ are known, Appendix EC.1 of Ankenman et al. (2010) shows that the MSE-optimal predictor of $\mathrm{Y}\left(\mathbf{x}_{0}\right)$ is given by

$$
\widehat{\mathrm{Y}}\left(\mathbf{x}_{0}\right)=\mathbf{f}\left(\mathbf{x}_{0}\right)^{\top} \beta+\Sigma_{\mathrm{M}}\left(\mathbf{x}_{0}, \cdot\right)^{\top} \Sigma^{-1}(\overline{\mathscr{Y}}-\mathbf{F} \beta),
$$

and the corresponding MSE is

$$
\operatorname{MSE}\left(\widehat{\mathrm{Y}}\left(\mathbf{x}_{0}\right)\right)=\Sigma_{\mathrm{M}}\left(\mathbf{x}_{0}, \mathbf{x}_{0}\right)-\Sigma_{\mathrm{M}}\left(\mathbf{x}_{0}, \cdot\right)^{\top} \Sigma^{-1} \Sigma_{\mathrm{M}}\left(\mathbf{x}_{0}, \cdot\right),
$$

where $\Sigma=\Sigma_{\mathrm{M}}+\Sigma_{\varepsilon}$ and $\mathbf{F}=\left(\mathbf{f}\left(\mathbf{x}_{1}\right)^{\top}, \mathbf{f}\left(\mathbf{x}_{2}\right)^{\top}, \ldots, \mathbf{f}\left(\mathbf{x}_{k}\right)^{\top}\right)^{\top}$. Let the $k \times k$ matrix $\Sigma_{\mathrm{M}}$ be the spatial variance-covariance matrix across the design points, i.e., its $(i, \ell)$ th entry is given by $\operatorname{Cov}\left(\mathrm{M}\left(\mathbf{x}_{i}\right), \mathrm{M}\left(\mathbf{x}_{\ell}\right)\right)$ as specified in (2); also let the $k \times 1$ vector $\Sigma_{\mathrm{M}}\left(\mathbf{x}_{0}, \cdot\right)$ record the spatial covariances between the $k$ design points and prediction point $\mathbf{x}_{0}$. Without the use of CRN across the design points (it is shown by Chen et al. 2012 that the use of CRN may degrade the predictive performance of SK), $\Sigma_{\varepsilon}$ becomes the $k \times k$ diagonal matrix whose $i$ th diagonal entry is given by $\mathrm{V}\left(\mathbf{x}_{i}\right) / n_{i}, i=1,2, \ldots, k$.

To apply (4) and (5) for mean response prediction, one must perform parameter estimation first. We start with estimating $\mathrm{V}\left(\mathbf{x}_{i}\right)$ in $\Sigma_{\varepsilon}$ by the sample variance $\left(n_{i}-1\right)^{-1} \sum_{j=1}^{n_{i}}\left(\mathscr{Y}_{j}\left(\mathbf{x}_{i}\right)-\overline{\mathscr{Y}}\left(\mathbf{x}_{i}\right)\right)^{2}$ for $i=1,2, \ldots, k$ to obtain $\widehat{\Sigma}_{\varepsilon}$, and substituting $\widehat{\Sigma}_{\varepsilon}$ into $\Sigma=\Sigma_{\mathrm{M}}+\Sigma_{\varepsilon}$. Then $\beta, \theta$, and $\tau^{2}$ can be obtained through maximum likelihood estimation (see, e.g., Ankenman et al., 2010; Chen and Kim, 2014) or alternative methods discussed in Section 5.1 of Kleijnen (2017). Finally, prediction via SK can be performed by substituting the resulting parameter estimates into (4) and (5). We note that with the parameter estimates plugged in, (4) becomes a nonlinear predictor and (5) tends to underestimate the true MSE. Nevertheless, such an approach does not seem to be causing serious problems in practice (Mehdad and Kleijnen, 2015). Finally, we will re-examine the analytical results derived via numerical studies with all the parameters being estimated in Section 5.

\section{Some Properties of Stochastic Kriging}

In this section we first present some results regarding the integrated mean squared error (IMSE) and the MSE of prediction achieved by SK in a sequential design setting; we then propose some sequential design strategies to implement metamodeling SK techniques. 


\subsection{Results on IMSE reduction achieved by stochastic kriging}

Assumption 1. The random field $\mathrm{M}$ is a stationary Gaussian random field, and $\varepsilon_{1}\left(\mathbf{x}_{i}\right), \varepsilon_{j}\left(\mathbf{x}_{i}\right), \ldots$ are i.i.d. $\mathscr{N}\left(0, \mathrm{~V}\left(\mathbf{x}_{i}\right)\right)$, independent of $\varepsilon_{j}\left(\mathbf{x}_{h}\right)$ for all $j$ and $h \neq i$ (i.e., no $C R N)$, and independent of $\mathrm{M}$.

Under Assumption 1, it is shown by Ankenman et al. (2010) that the SK predictor (4) is the conditional expectation of $\mathrm{Y}\left(\mathbf{x}_{0}\right)$ given $\bar{Y}$ with known parameters $\tau^{2}, \theta, \beta$ and intrinsic variance-covariance matrix $\Sigma_{\varepsilon}$, and it is the minimum MSE predictor (Appendix EC.1 of Ankenman et al., 2010). We note that this assumption may fail to hold if the SK metamodel specified by (1) is not adequate for describing the relationship between the simulation outputs and the true mean response surface of interest. In such a situation, one may want to perform metamodel validation (Kleijnen, 2015) or investigate the consequences of applying SK with biased sample estimates (Chen and Kim, 2014). In the rest of this paper, however, Assumption 1 is always considered to be fulfilled.

Define the integrated mean squared error (IMSE) achieved by SK over a compact set $\mathscr{X} \in \mathbb{R}^{d}$ as

$$
\operatorname{IMSE}=\int_{\mathscr{X}} \operatorname{MSE}\left(\widehat{\mathrm{Y}}\left(\mathbf{x}_{0}\right)\right) d \mathbf{x}_{0}
$$

where $\operatorname{MSE}\left(\widehat{Y}\left(\mathbf{x}_{0}\right)\right)$ is given by (5). Given a design consisting of $k$ existing design points with $n_{i}$ simulation replications allocated to the $i$ th design point $\mathbf{x}_{i}$, $i=1,2, \ldots, k$, the following result quantifies the change in IMSE, $\Delta \mathrm{IMSE}^{o}$ resulting from assigning additional $\Delta n$ replications to the $k$ existing design points, i.e., $\Delta \mathrm{IMSE}^{o}=\mathrm{IMSE}_{k}^{\Delta}-\mathrm{IMSE}_{k}$, where the subscript indicating the size of the design-point set under consideration.

Theorem 1. Suppose that Assumption 1 holds and that $\tau^{2}, \theta$ and $\beta$ and $\Sigma_{\varepsilon}$ are known. Assume that $n_{i}$ replications have been allocated at $\mathbf{x}_{i}$ for $i=1,2, \ldots, k$. If $\Delta n$ additional simulation replications are assigned to these $k$ design points such that $\sum_{i=1}^{k} \Delta n_{i}=\Delta n$, then the IMSE decreases and $\Delta \mathrm{IMSE}^{o}:=\mathrm{IMSE}_{k}^{\Delta}-\mathrm{IMSE}_{k} \leq$ 0 , where $\mathrm{IMSE}_{k}^{\Delta}$ denotes the IMSE obtained as a result of the allocation. Furthermore, let $\Delta \Sigma_{\varepsilon}=\operatorname{diag}\left\{C_{1}, C_{2}, \ldots, C_{k}\right\}$ where $C_{i}=\frac{\mathrm{V}\left(\mathbf{x}_{i}\right)}{n_{i}+\Delta n_{i}}-\frac{\mathrm{V}\left(\mathbf{x}_{i}\right)}{n_{i}}=\frac{-\mathrm{V}\left(\mathbf{x}_{i}\right) \Delta n_{i}}{\left(n_{i}+\Delta n_{i}\right) n_{i}}$ for $i=1,2, \ldots, k$. If $\max _{i=1, \ldots, k} \Delta \Sigma_{\varepsilon}(i, i) \ll 1$ such that $\mathbf{I}_{k}+\Delta \Sigma_{\varepsilon} \Sigma^{-1} \approx \mathbf{I}_{k}$ (the $k \times k$ identity matrix), then the optimal budget allocation rule that leads to the maximum reduction in IMSE is to assign $\Delta n_{i}^{*}$ replications to $\mathbf{x}_{i}$ such that

$$
n_{i}+\Delta n_{i}^{*} \propto \sqrt{Q_{i} \bigvee\left(\mathbf{x}_{i}\right)}, i=1,2, \ldots, k,
$$


where $Q_{i}:=\tau^{4} \mathbf{1}_{k}^{\top}\left(\mathbf{W}_{k} \circ\left[\Sigma^{-1} \mathbf{J}^{(i i)} \Sigma^{-1}\right]\right) \mathbf{1}_{k}$, ○ is the operator for the Hadamard product of matrices, $\mathbf{J}^{(i i)}$ is the $k \times k$ matrix with one in the ith diagonal entry and zeros elsewhere, and $\mathbf{1}_{k}$ denotes the $k \times 1$ vector of ones; $\mathbf{W}_{k}$ denotes the $k \times k$ matrix whose $(i, j)$ th entry is given by $\int_{\mathscr{X}} r_{0 i} r_{0 j} d \mathbf{x}_{0}$, for $i, j=1,2, \ldots, k$, and $r_{0, i}=\mathscr{R}\left(\mathbf{x}_{0}-\mathbf{x}_{i} ; \theta\right), i=1,2, \ldots, k$.

The proof of Theorem 1 is given in AppendixA. We can see from (7) that the allocation of additional replications tries to adjust the simulation budget assigned to an existing design point proportional to a term of the simulation standard deviation at that design point weighted by some spatial correlation structure. Therefore, both sampling uncertainty and response surface uncertainty matter in the budget allocation.

We note that the integrated mean squared error (IMSE) based criteria are often used for constructing experimental designs such that the resulting metamodel can achieve satisfactory predictive performance across the design space. For kriging prediction with nugget effect, Gratiet and Garnier (2015) provide theoretical results on the asymptotic values of pointwise MSE and IMSE and obtain the convergence rates of IMSE for selected kernels as the number of design points increases to infinity; they further discuss how the theoretical results can be used to determine the total budget required to achieve a pre-specified accuracy level in terms of IMSE. The differences between their work and our results given in Theorem 1 lie on the following aspects. First, in deriving the results they assume that the simulation variance is homogeneous across the design space and that its magnitude is proportional to the number of design points used, whereas we do not need such an assumption. Second, their theoretical analysis is facilitated by assuming that the total budget (the total number of observations) tends to infinity, and the type of experimental designs that directly benefit from their theoretical results are those "one-shot" designs with a large total budget to expend and with the same number of simulation replications being allocated to all design points. In contrast, our results are helpful for constructing sequential design strategies to expend a fixed simulation budget, with a particular emphasis on seeking efficient budget allocation rules that aim at reducing the impact of heteroscedasticity on the predictive performance of SK.

Given the existing set of $k$ design points with $n_{i}$ simulation replications allocated to $\mathbf{x}_{i}, i=1,2, \ldots, k$, the following result is about the change in IMSE, $\Delta \mathrm{IMSE}^{n}$, resulting from assigning $\Delta n$ replications to a new design point, say, $\mathbf{x}_{k+1}$; that is, $\Delta \operatorname{IMSE}\left(\mathbf{x}_{k+1} ; \Delta n\right)=\mathrm{IMSE}_{k+1}-\mathrm{IMSE}_{k}$; here $\mathrm{IMSE}_{k+1}$ denotes the IMSE obtained upon adding $\Delta n$ replications at $\mathbf{x}_{k+1}$. 
Theorem 2. Suppose that Assumption 1 holds and that $\tau^{2}, \theta, \beta$ and $\Sigma_{\varepsilon}$ are known. Assume that $n_{i}$ replications have been allocated at $\mathbf{x}_{i}$ for $i=1,2, \ldots, k$. If $\Delta n$ simulation replications are assigned to a new design point $\mathbf{x}_{k+1}$, then the IMSE decreases and

$$
\Delta \operatorname{IMSE}\left(\mathbf{x}_{k+1} ; \Delta n\right):=\operatorname{IMSE}_{k+1}-\operatorname{IMSE}_{k}=-\tau^{4} \ell \mathbf{1}_{k+1}^{\top}\left[\mathbf{W}_{k+1} \circ \mathbf{E}\right] \mathbf{1}_{k+1} \leq 0,
$$

where

$$
\mathbf{W}_{k+1}=\left(\begin{array}{cc}
w_{k+1, k+1} & \mathbf{w}_{k+1, \cdot}^{\top} \\
\mathbf{w}_{k+1, \cdot} & \mathbf{W}_{k}
\end{array}\right)
$$

$w_{k+1, k+1}=\int_{\mathscr{X}} r_{k+1,0}^{2} d \mathbf{x}_{0}, \mathbf{w}_{k+1, \cdot}=\left(\int_{\mathscr{X}}\left(r_{k+1,0} \cdot r_{0,1}\right) d \mathbf{x}_{0}, \ldots, \int_{\mathscr{X}}\left(r_{k+1,0} \cdot r_{0, k}\right) d \mathbf{x}_{0}\right)^{\top}$, with $r_{i, j}=\mathscr{R}\left(\mathbf{x}_{i}-\mathbf{x}_{j} ; \boldsymbol{\theta}\right)$ for $i, j=0,1, \ldots, k+1 ; \ell=\left(\operatorname{MSE}_{k}\left(\mathrm{Y}\left(\mathbf{x}_{k+1}\right)\right)+\frac{\mathrm{V}\left(\mathbf{x}_{k+1}\right)}{\Delta n}\right)^{-1}$ is a scalar, and $\mathrm{MSE}_{k}\left(\mathrm{Y}\left(\mathbf{x}_{k+1}\right)\right)$ is simply the MSE obtained in predicting at the $(k+1)$ th design point by using the existing set of $k$ design points;

$$
\mathbf{E}=\left(\begin{array}{cc}
1 & -\tau^{2} \mathbf{r}_{k+1, \cdot}^{\top}{ }^{\Sigma_{k}^{-1}} \\
-\tau^{2} \Sigma_{k}^{-1} \mathbf{r}_{k+1, \cdot} & \tau^{4} \Sigma_{k}^{-1} \mathbf{r}_{k+1, \cdot} \mathbf{r}_{k+1, \cdot}^{\top} \Sigma_{k}^{-1}
\end{array}\right)
$$

$\mathbf{r}_{k+1, \cdot}$ denotes the $k \times 1$ vector $\mathbf{r}_{k+1, \cdot}=\left(r_{k+1,1}, \ldots, r_{k+1, k}\right)^{\top}$, and $\Sigma_{k}=\Sigma_{\mathrm{M}, k}+\Sigma_{\varepsilon, k}$, and $k$ in the subscript indicates the size of the existing design-point set.

The proof of Theorem 2 is available in AppendixB. The expression of $\Delta \operatorname{IMSE}\left(\mathbf{x}_{k+1} ; \Delta n\right)$ reveals that it is preferred that $\mathbf{x}_{k+1}$ has strong spatial correlations with a majority of the prediction points. Furthermore, the lower the sampling variability at $\mathbf{x}_{k+1}$ is, the greater $\Delta \mathrm{IMSE}\left(\mathbf{x}_{k+1} ; \Delta n\right)$ becomes.

\subsection{A result on $\mathrm{MSE}$ at an existing design point}

Suppose that the existing design set consists of $k$ design points with $n_{i}$ simulation replications allocated to $\mathbf{x}_{i}, i=1,2, \ldots, k$. The following result can be obtained regarding the MSE of prediction at each existing design point.

Lemma 1. Suppose that Assumption 1 holds and that $\tau^{2}, \theta, \beta$ and $\Sigma_{\varepsilon}$ are known. Assume that $n_{i}$ replications have been allocated at $\mathbf{x}_{i}$ for $i=1,2, \ldots, k$. Then, the following expression for $\operatorname{MSE}\left(\widehat{\mathrm{Y}}\left(\mathbf{x}_{i}\right)\right)$ holds:

$$
\frac{1}{\operatorname{MSE}\left(\widehat{\mathrm{Y}}\left(\mathbf{x}_{i}\right)\right)}=\frac{1}{\operatorname{MSE}_{-i}\left(\widehat{\mathrm{Y}}_{-i}\left(\mathbf{x}_{i}\right)\right)}+\frac{1}{\left(\mathrm{~V}\left(\mathbf{x}_{i}\right) / n_{i}\right)}, i=1,2, \ldots, k,
$$

where $\operatorname{MSE}_{-i}\left(\widehat{Y}_{-i}\left(\mathbf{x}_{i}\right)\right)$ denotes the MSE of prediction obtained by predicting at $\mathbf{x}_{i}$ using the outputs collected at all the other design points but $\mathbf{x}_{i}$. 
The proof of Lemma 1 is similar to that is given for Equation (13) by Kamiński (2015), and hence is omitted for brevity.

Remark 1. The following observations made from Lemma 1 shed some light on an adaptive exploration and exploitation rule that can be useful in a sequential design setting:

(i) From the right-hand side of (9), we can see that two sources of uncertainty contribute to the MSE of prediction at $\mathbf{x}_{i}$, namely, the response-surface uncertainty derived from prediction based on the other $k-1$ design points, $\operatorname{MSE}_{-i}\left(\widehat{Y}_{-i}\left(\mathbf{x}_{i}\right)\right)$, and the uncertainty arising from estimation at the design point itself, $\mathrm{V}\left(\mathbf{x}_{i}\right) / n_{i}$.

(ii) $\operatorname{MSE}\left(\widehat{\mathrm{Y}}\left(\mathbf{x}_{i}\right)\right)$ is upper bounded by the intrinsic variance at $\mathbf{x}_{i}, \mathrm{~V}\left(\mathbf{x}_{i}\right) / n_{i}$. Therefore, the prediction result given by SK improves the estimation result obtained by sampling at a design point.

(iii) For a given design point, say, $\mathbf{x}_{i}$, if the ratio $\frac{\operatorname{MSE}\left(\widehat{\mathrm{Y}}\left(\mathbf{x}_{i}\right)\right)}{\left(\mathrm{V}\left(\mathbf{x}_{i}\right) / n_{i}\right)}$ is very close to 1 , it implies that the uncertainty in predicting at $\mathbf{x}_{i}$ using the information from all the other existing design points is quite high and the most useful information for prediction at $\mathbf{x}_{i}$ comes from sampling at that location, hence an indication that a new design point in the neighborhood of $\mathbf{x}_{i}$ can be desirable to further increase the predictive accuracy at $\mathbf{x}_{i}$. On the other hand, if the ratio $\frac{\operatorname{MSE}\left(\widehat{Y}\left(\mathbf{x}_{i}\right)\right)}{\left(\mathrm{V}\left(\mathbf{x}_{i}\right) / n_{i}\right)}$ is very close to 0 , it implies that the sampling uncertainty at $\mathbf{x}_{i}$ is very high, hence an indication that adding more simulation budget at $\mathbf{x}_{i}$ can be desirable to further increase the predictive accuracy at $\mathbf{x}_{i}$.

Now let us consider the set of such ratios obtained at the $k$ existing design points, $\left\{\frac{\operatorname{MSE}\left(\widehat{Y}\left(\mathbf{x}_{i}\right)\right)}{\mathrm{V}\left(\mathbf{x}_{i}\right) / n_{i}}\right\}_{i=1}^{k}$. If a low quantile, say, the $\alpha$-quantile of this set of ratios (e.g., $\alpha=0.1$ ) is close to 1 , the discussion above indicates that to make prediction at a majority of the $k$ existing design points, not as much can be learned from other existing design points than sampling at themselves. That is, response-surface uncertainty dominates sampling uncertainty at a majority of the $k$ design points and there is a need to add a new design point in the next iteration. On the other hand, if a high quantile, say, $\beta$ quantile of the set of ratios (say, $\beta=0.9$ ) is close to 0 , it indicates that to predict at a majority of the $k$ existing design points, the sampling uncertainty at themselves is too high to provide useful information to make accurate prediction at these design points. That is, sampling uncertainty dominates response-surface uncertainty and there is a need to allocate more simulation 
budget at the existing design points to reduce sampling uncertainty in the next iteration.

\section{Sequential Design Strategies for Stochastic Kriging}

With a total computational budget of, say $N$, simulation replications to allocate, the goal of a design strategy in this paper is to sequentially add design points and to allocate simulation budget to each of them, such that the resulting SK metamodel can achieve as high predictive accuracy over $\mathscr{X}$ as possible when the budget is exhausted. To simplify our analysis, suppose that there is a relatively dense candidate design-point set, $\mathbf{X}^{k_{\mathrm{tot}}}$, consisting of $k_{\mathrm{tot}}$ distinct points in $\mathscr{X}$, from which we consider selecting design points to run simulations. Notice that the value of $k_{\text {tot }}$ should be large enough so that the $k_{\text {tot }}$ design points in $\mathbf{X}^{k_{\text {tot }}}$ can cover the design space $\mathscr{X}$ adequately; in fact, we recommend to use $k_{\text {tot }}>10 d$ where $d$ is the dimension of the design space based on the suggestion given by Loeppky et al. (2009).

A sequential design strategy typically begins with a pilot experiment (i.e., the first iteration of a sequential experiment). The pilot experiment is conducted at $k_{0}$ points chosen from $\mathbf{X}^{k_{\text {tot }}}$ following a space-filling design with the $i$ th point receiving $n$ simulation replications $\left(k_{0} n<N\right)$. Denote the pilot design by $\mathscr{D}_{1}=$ $\left\{\left(\mathbf{x}_{i}, n\right), i=1,2, \ldots, k_{0}\right\}$ and the initial design-point set by $\mathbf{S}_{1}=\left\{\mathbf{x}_{i}, i=1,2, \ldots, k_{0}\right\} ;$ hence $\left|S_{1}\right|=k_{0}$. The purpose of this pilot experiment is to collect some information about the underlying response surface and to have an initial assessment of the sampling variability across $\mathscr{X}$. The resulting data can be used to fit an initial SK metamodel and a simulation variance metamodel across $\mathscr{X}$. Define $\Delta n$ as the step size or the number of replications to allocate on each iteration. On each of the subsequent iterations, we choose either a new design point or some existing ones from $\mathbf{X}^{k_{\text {tot }}}$ and assign $\Delta n$ replications according to the design strategy adopted. To make sure that the pilot design set is a proper subset of the candidate design set, namely, $\mathbf{S}_{1} \subset \mathbf{X}^{k_{\mathrm{tot}}}$, and that both design sets have the space-filling property, we consider using nested Latin hypercube design (Qian, 2009) or alternative designs such as sliced orthogonal array-based Latin hypercube design (Hwang et al., 2016) for constructing these two design sets.

We next propose three sequential design strategies based on the results given in Section 3. All these strategies are constructed to sequentially reduce the IMSE, which directly quantifies the predictive accuracy achieved by the fitted SK metamodel built on a given design. Denote the experiment design generated on the $j$ th iteration by $\mathscr{D}_{j}$ and the corresponding design-point set by $\mathbf{S}_{j}, j \geq 1$. 
1. IMSE-based design strategy (IMSE). On iteration $j$ for $j \geq 2$, this strategy chooses the next point from the candidate design-point set $\mathbf{X}^{k_{\text {tot }}}$ that leads to the minimum IMSE of prediction. That is, $\mathbf{x}^{*}:=\operatorname{argmin}_{\mathbf{x} \in \mathbf{X}^{k_{\text {tot }}}}$ IMSE, where IMSE is obtained based on the candidate design $\left\{\mathscr{D}_{j-1} \bigcup(\mathbf{x}, \Delta n)\right\}$ for each $\mathbf{x} \in \mathbf{X}^{k_{\mathrm{tot}}}$; the resulting $\mathbf{x}^{*}$ can be either a new or an existing design point. Then set $\mathscr{D}_{j}=\mathscr{D}_{j-1} \bigcup\left(\mathbf{x}^{*}, \Delta n\right)$ and $\mathbf{S}_{j}=\mathbf{S}_{j-1} \bigcup \mathbf{x}^{*}$.

2. Stepwise Approximate Optimal Design (SAO). On iteration $j$ for $j \geq 2$, calculate the possible changes in IMSE, $\triangle \mathrm{IMSE}^{o}$ and $\Delta \mathrm{IMSE}^{n}$, respectively, obtained by allocating $\Delta n$ runs to all the existing design points in $\mathbf{S}_{j-1}$ following the allocation rule given by (7), and by assigning $\Delta n$ simulation runs to one candidate design point in $\mathbf{X}^{k_{\text {tot }}} \backslash \mathbf{S}_{j-1}$. Specifically,

- To estimate $\Delta \mathrm{IMSE}^{o}$, we first find $\Delta n_{i}$ to assign to each existing design point in $\mathbf{S}_{j-1}$ by using

$$
\Delta n_{i}=\max \left\{0, \frac{\sqrt{Q_{i} \vee\left(\mathbf{x}_{i}\right)}}{\sum_{i: \mathbf{x}_{i} \in \mathbf{S}_{j-1}} \sqrt{Q_{i} \vee\left(\mathbf{x}_{i}\right)}} \times\left(\sum_{i: \mathbf{x}_{i} \in \mathbf{S}_{j-1}} n_{i}+\Delta n\right)-n_{i}\right\},
$$

where $n_{i}$ is the number of runs that has been already assigned to design point $\mathbf{x}_{i}, \forall \mathbf{x}_{i} \in \mathbf{S}_{j-1}$.

- To estimate $\Delta \mathrm{IMSE}^{n}$, we have

$$
\Delta \mathrm{IMSE}^{n}=\max _{\mathbf{x} \in \mathbf{X}^{k_{\text {tot }}} \backslash \mathbf{S}_{j-1}} \Delta \operatorname{IMSE}(\mathbf{x} ; \Delta n) .
$$

Therefore, for the $j$ th iteration, if $\Delta \mathrm{IMSE}^{o}>\Delta \mathrm{IMSE}^{n}$, then allocate $\Delta n$ runs to the existing design points in $\mathbf{S}_{j-1}$ following the allocation rule given by (10), and set $\mathscr{D}_{j}=\mathscr{D}_{j-1} \bigcup\left\{\left(\mathbf{x}_{i}, \Delta n_{i}\right), \forall \mathbf{x}_{i} \in \mathbf{S}_{j-1}\right\}$ and $\mathbf{S}_{j}=\mathbf{S}_{j-1}$; otherwise, assign $\Delta n$ replications to a new design point $\mathbf{x}^{*}$, where

$$
\mathbf{x}^{*}=\underset{\mathbf{x} \in \mathbf{X}^{k_{\text {tot }} \backslash \mathbf{S}_{j-1}}}{\operatorname{argmax}} \Delta \operatorname{IMSE}(\mathbf{x} ; \Delta n) ;
$$

and set $\mathscr{D}_{j}=\mathscr{D}_{j-1} \bigcup\left(\mathbf{x}^{*}, \Delta n\right)$ and $\mathbf{S}_{j}=\mathbf{S}_{j-1} \bigcup \mathbf{x}^{*}$.

3. Balanced Stepwise Approximate Optimal Design strategy (BSAO). Everything is the same as for SAO, but this strategy adaptively balances exploration and exploitation (i.e., reducing response-surface uncertainty vs. sampling uncertainty) according to the following transition rule inspired by Lemma 1 and Remark 1 (iii): Calculate the set of ratios $\left\{R_{i}: R_{i}=\frac{\operatorname{MSE}\left(\widehat{\mathrm{Y}}\left(\mathbf{x}_{i}\right)\right)}{\mathrm{V}\left(\mathbf{x}_{i}\right) / n_{i}}\right.$, $\left.\forall \mathbf{x}_{i} \in \mathbf{S}_{j-1}\right\}$, 
- if the $\alpha$-quantile (say, 0.1-quantile) of $R_{i}$ 's is larger than $c_{1}$, then assign $\Delta n$ runs at $\mathbf{x}^{*}$ as defined by (11);

- if the $\beta$-quantile (say, 0.9-quantile) of $R_{i}$ 's is smaller than $c_{2}$, then assign $\Delta n_{i}$ replications as given by (10) to $\mathbf{x}_{i} \in \mathbf{S}_{j-1}, \forall \mathbf{x}_{i} \in \mathbf{S}_{j-1}$.

Furthermore, update $\mathscr{D}_{j}$ and $\mathbf{S}_{j}$ correspondingly as described for SAO.

In the numerical experiments performed in Section 5, $\alpha=0.1$ and $\beta=0.9$ and $c_{1}=c_{2}=0.5$ are used.

Remark 2. To implement the aforementioned sequential design strategies, an estimate of the simulation variance $\mathrm{V}(\mathbf{x})$ at $\mathbf{x} \in \mathscr{X}$ is required. To this end, a kriging metamodel for the logarithm transformed simulation variance function, $\ln (\mathrm{V}(\cdot))$, is built using the log-transformed sample variances at all the existing design points. Such a transformation ensures the non-negativity of the resulting variance estimates, in contrast to building a kriging model directly for $\mathrm{V}(\cdot)$ as done by Ankenman et al. (2010). The variance predictor of $\mathrm{V}\left(\mathbf{x}_{0}\right)$ then can be obtained as $\left.\exp \left\{\ln \widehat{\left(\mathrm{V}\left(\mathbf{x}_{0}\right)\right.}\right)\right\}$ where $\ln \widehat{\left(\mathrm{V}\left(\mathbf{x}_{0}\right)\right)}$ is the kriging predictor. We note that such an approach has also been considered by Kleijnen (2017) and Kamiński (2015); other methods for variance function estimation can be adopted as well, but the aforementioned approach is implemented in the numerical examples given in Section 5.

\section{Numerical Examples}

In this section we demonstrate the predictive performances of SK with the proposed sequential design strategies implemented through three illustrative examples with distinct features. For all the examples considered, assuming that little is known about the true response surface, we adopt the recommendation in the kriging literature to model the performance-measure response surface as $\mathrm{Y}(\mathbf{x})=\beta_{0}+\mathrm{M}(\mathbf{x})$. The parameter estimates $\widehat{\beta}_{0}, \widehat{\tau}^{2}$, and $\widehat{\theta}$ are obtained through maximum likelihood estimation. The MATLAB code for standard SK can be found at www. stochastickriging. net; and the source code used in this section is available upon request.

\subsection{A One-Dimensional Example}

The goal of this section is to compare the performances of SK delivered by adopting different sequential designs to estimate the function of interest $Y(x)$ over 
a one-dimensional design space $\mathscr{X}$, given a total budget of $N$ simulation replications to expend.

A sequential experiment starts with a pilot design $\mathscr{D}_{1}$ consisting of 7 equally spaced design points with $n=20$ simulation runs assigned to each one of them. On each subsequent iteration, $\Delta n=20$ replications are allocated to design point(s) selected from the set of potential design points, $\mathbf{X}^{k^{\mathrm{tot}}}$, which consists of $k_{\text {tot }}=193$ equally spaced points in $\mathscr{X}$. A testing set $\mathbf{X}^{K}$ with $K$ (typically $K \geq k_{\text {tot }}$ ) test points densely located in $\mathscr{X}$ is constructed to evaluate the predictive performances of SK. Notice that $\mathbf{X}^{k_{\text {tot }}} \subseteq \mathbf{X}^{K}$; here for simplicity we set them equal to each other.

The entire experiment is repeated for 100 independent macro-replications, and the corresponding performance measure, the empirical root mean squared errors (ERMSE), is calculated as follows,

$$
\operatorname{ERMSE}_{\ell}=\sqrt{\frac{1}{K} \sum_{i=1}^{K}\left(\widehat{\mathrm{Y}}_{\ell}\left(x_{i}\right)-\mathrm{Y}\left(x_{i}\right)\right)^{2}}, \quad \ell=1,2, \ldots, 100
$$

where $\widehat{Y}_{\ell}(\cdot)$ represents the prediction given by SK on the $\ell$ th macro-replication.

It is known that the steady-state mean number of customers in an $\mathrm{M} / \mathrm{M} / 1$ queue with service rate 1 and arrival rate $x$ is $\mathrm{Y}(x)=x /(1-x)$, for $x \in(0,1)$. If a simulation replication is initiated at time 0 in steady state given the arrival rate $x$, then the average number of customers in system during the first $T$ time units is an unbiased estimator for $\mathrm{Y}(x)$, and its variance is approximately $2 x(1+x) /(T(1-$ $x)^{4}$ ) for large $T$ (Whitt, 1989). We consider the following example inspired by the $\mathrm{M} / \mathrm{M} / 1$ example given by Ankenman et al. (2010). The simulated response on each replication at a point $x$ is generated as $\mathscr{Y}_{j}(x)=\mathrm{Y}(x)+\varepsilon_{j}(x)$, with $\varepsilon_{j}(x)$ 's being i.i.d. $\mathscr{N}(0, \mathrm{~V}(x))$ random variables, where

$$
\mathrm{Y}(x)=x /(1-x), \quad \mathrm{V}(x)=2 x(1+x) /\left(T(1-x)^{4}\right),
$$

with $x \in[0.3,0.9]$ and $T=1000$. The mean and variance functions are shown in Figures 1 (a) and (b). Notice that the simulation variance increases explosively as $x$ increases which makes the prediction task considerably challenging.

Results. Table 1 summarizes the ERMSEs obtained using different sequential design strategies with a total budget of $N=500$ and 1000 simulation replications. We observe that IMSE is the least efficient design strategy in terms of the ERMSEs achieved by the end of sequential iterations, and SAO and BSAO outperform IMSE by a large margin for this $\mathrm{M} / \mathrm{M} / 1$ inspired example with BSAO performing a little better than SAO. Figure 2 (a) provides a closer look at the 


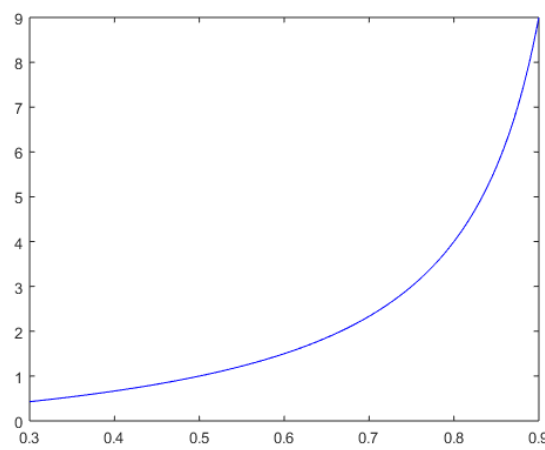

(a) $\mathrm{Y}(x)$

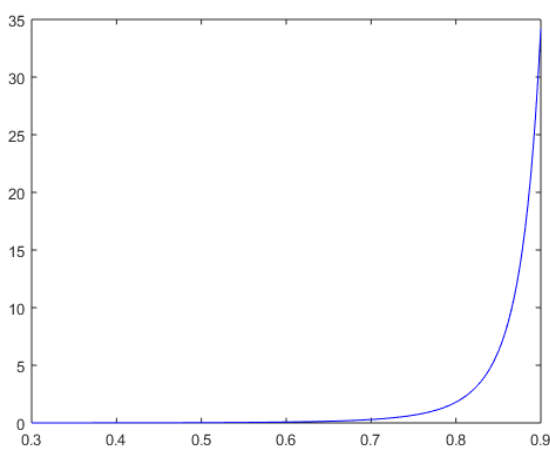

(b) $\mathrm{V}(x)$

Figure 1: The mean and variance functions for the $\mathrm{M} / \mathrm{M} / 1$ inspired example.

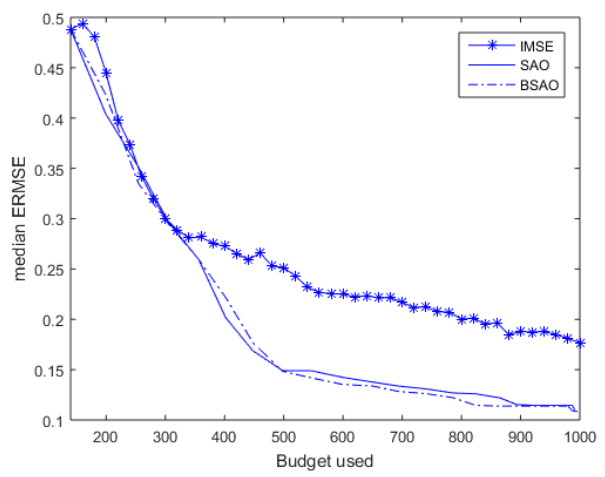

(a) Median ERMSE

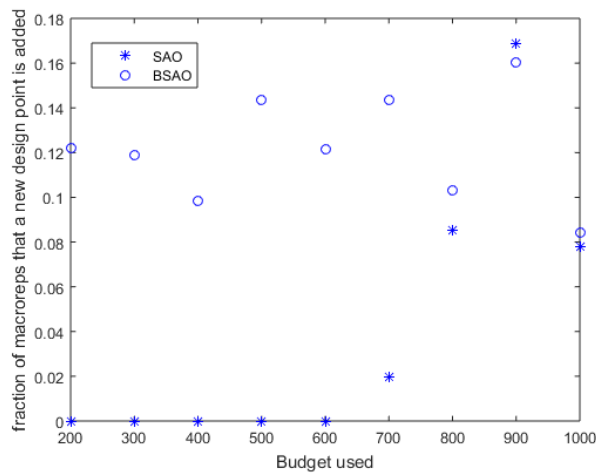

(b) Fraction of macro-reps that a new design point is added

Figure 2: The M/M/1 inspired example with $N=1000$. Figure 2(a) plots the median ERMSE of 100 macro-replications achieved by IMSE, SAO and BSAO. Figure 2(b) plots the fractions of 100 macro-replications that a new design point is added by SAO and BSAO, respectively. 


\begin{tabular}{|c|c|c|c|c|c|c|c|}
\hline \hline \multicolumn{4}{|c|}{$N=500$} & \multicolumn{4}{c|}{$N=10^{3}$} \\
\hline quartiles & IMSE & SAO & BSAO & quartiles & IMSE & SAO & BSAO \\
\hline 25th & 0.173 & 0.116 & 0.114 & 25th & 0.133 & 0.094 & 0.084 \\
\hline 50th & 0.233 & 0.151 & 0.150 & 50th & 0.177 & 0.110 & 0.108 \\
\hline 75th & 0.326 & 0.269 & 0.226 & 75th & 0.253 & 0.139 & 0.132 \\
\hline
\end{tabular}

Table 1: Summary statistics of ERMSEs obtained with $n=20, \Delta n=20$ and varying total simulation budgets $N$ for the $\mathrm{M} / \mathrm{M} / 1$ inspired example

\begin{tabular}{|c|c|c|c|c|c|c|c|}
\hline \hline \multicolumn{4}{|c|}{$N=500$} & \multicolumn{4}{c|}{$N=10^{3}$} \\
\hline & IMSE & SAO & BSAO & & IMSE & SAO & BSAO \\
\hline min & 15 & 7 & 7 & min & 31 & 7 & 8 \\
\hline median & 19 & 7 & 8 & median & 30 & 8 & 9 \\
\hline mean & 19 & 7 & 8 & mean & 30 & 8 & 9 \\
\hline max & 22 & 8 & 13 & max & 37 & 8 & 13 \\
\hline
\end{tabular}

Table 2: A summary of the total numbers of design points used by different sequential design strategies with $n=20, \Delta n=20$ and varying total simulation budgets $N$ for the $\mathrm{M} / \mathrm{M} / 1$ inspired example

predictive performances in terms of the respective median ERMSEs achieved by IMSE, SAO and BSAO across the 100 macro-replications. We see that with a little budget expended at the beginning, the performances due to the three design strategies do not differ much; but as more and more budget is expended, SAO and BSAO start to outperform IMSE and the performance gap persists until the total budget is exhausted. It is also observed in Figure 2 (a) that BSAO gives a slightly lower median ERMSE than SAO, especially as more budget is consumed. The reason is that BSAO improves over SAO by adaptively balancing the need of reducing the sampling uncertainty at the existing design points and the desire of adding a new design point in a less explored region to further reduce the responsesurface uncertainty.

Figure 2 (b) plots the respective fractions of times out of the 100 macroreplications that $\mathrm{SAO}$ and $\mathrm{BSAO}$ have a new design point added with the budget consumed. It is evident that BSAO is more likely to add a new design point than SAO in the early stage of the budget allocation process which results in a better predictive performance in the end. On the other hand, IMSE tends to spend the budget more aggressively on adding new design points, which is manifested by the total numbers of design points added by the three strategies summarized in 
Table 2 over 100 macro-replications; nevertheless, this practice of IMSE does not prove to be an effective one to use for the $\mathrm{M} / \mathrm{M} / 1$ inspired example, where the significant impact of simulation variance deserves careful consideration.

\subsection{A Two-Dimensional Example}

We consider using nested Latin hypercube design (see Qian (2009)) as our candidate design-point set; alternative designs include sliced orthogonal array-based Latin hypercube design; see Hwang et al. (2016) for more details. A two-layer nested Latin hypercube design (LHD) of $k_{\text {tot }}=100$ points in $\mathscr{X}$ is constructed as the full candidate design set $\mathbf{X}^{k_{\text {tot }}}$ : The first layer $\mathbf{X}^{k_{0}}$ is a LHD with $k_{0}=20$ points and the full set $\mathbf{X}^{k_{\text {tot }}}$ is a LHD with $k_{\text {tot }}$ points; and the name of the design follows from the fact that $\mathbf{X}^{k_{0}} \subset \mathbf{X}^{k_{\mathrm{tot}}}$. The initial design is conveniently chosen as $\mathbf{X}^{k_{0}}$, a LHD of $k_{0}$ points itself, with $n$ simulation runs allocated at each point. Therefore, in each iteration, according to a given design strategy, we consider selecting points from $\mathbf{X}^{k_{\text {tot }}}$ to allocate $\Delta n$ simulation runs.

To evaluate the predictive accuracy achieved by SK with the proposed sequential design strategies implemented, we construct the testing set $\mathbf{X}^{K}$ consisting of $K=900$ test points, i.e., a grid design of regularly spaced 900 points in $\mathscr{X}$. The entire experiment is repeated for 50 independent macro-replications, and the corresponding performance measure is ERMSE as defined by (12).

The 2-dimensional example considered here is adapted from the 2D-sine function used by Ajdari and Mahlooji (2014). The function to be approximated is given by

$$
\mathrm{Y}(\mathbf{x})=x_{1} \sin \left(\pi x_{2}\right)+x_{2} \sin \left(\pi x_{1}\right)
$$

where $\mathbf{x}=\left(x_{1}, x_{2}\right)^{\top}, x_{i} \in[-1,1]$ for $i=1,2$. The simulated response at a point $\mathbf{x}$ on the $j$ th replication is given by $\mathscr{Y}_{j}(\mathbf{x})=\mathrm{Y}(\mathbf{x})+\varepsilon_{j}(\mathbf{x})$, where $\varepsilon_{j}(\mathbf{x})$ 's are i.i.d. $\mathscr{N}(0, \mathrm{~V}(\mathbf{x}))$ random variables with $\mathrm{V}(\mathbf{x})=(|\mathrm{Y}(\mathbf{x})|+1)^{2}$. The contour plots of the mean and variance functions are given in Figure 3 (a) and (b), respectively. Notice that the simulation variance is a quadratic function of $|Y(\mathbf{x})|$. Since the function $\mathrm{Y}(\mathbf{x})$ changes its sign across the design space $\mathscr{X}=[-1,1] \times[-1,1]$, it is not an easy task to obtain accurate prediction results, despite that the variance function $\mathrm{V}(\mathbf{x})$ here does not change as dramatically as the one in the $M / M / 1$ inspired example.

Results. Table 3 summarizes the ERMSEs obtained using different sequential design strategies with a total budget of $N=1000$ simulation replications with two sets of values specified for $\Delta n$ and $n$. In both cases, SAO and BSAO are found to outperform IMSE with BSAO performing better than SAO. Furthermore, 


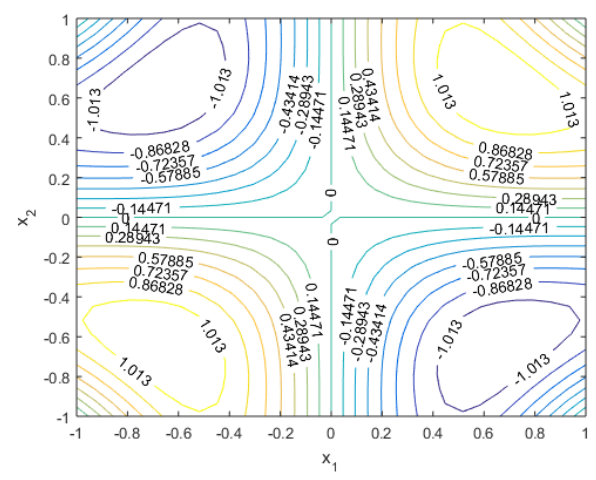

(a) $Y(\mathbf{x})$

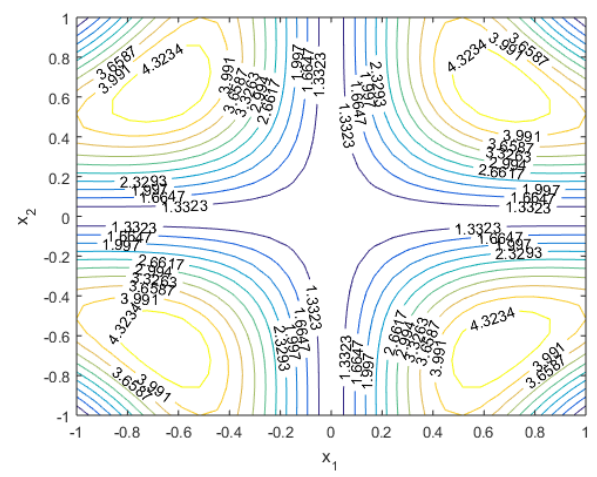

(b) $\mathrm{V}(\mathbf{x})$

Figure 3: Contour plots of true mean and variance functions for the 2D-Sine experiment.

\begin{tabular}{|c|c|c|c|c|c|c|c|}
\hline \hline \multicolumn{3}{|c|}{$n=10, \Delta n=10, N=10^{3}$} & \multicolumn{4}{c|}{$n=20, \Delta n=20, N=10^{3}$} \\
\hline quartiles & IMSE & SAO & BSAO & quartiles & IMSE & SAO & BSAO \\
\hline 25th & 0.208 & 0.198 & 0.199 & 25th & 0.203 & 0.197 & 0.191 \\
\hline 50th & 0.245 & 0.219 & 0.216 & 50th & 0.224 & 0.226 & 0.216 \\
\hline 75th & 0.254 & 0.248 & 0.246 & 75th & 0.251 & 0.256 & 0.242 \\
\hline
\end{tabular}

Table 3: Summary statistics of ERMSEs obtained for the 2D-Sine example

a comparison of the results for $\Delta n=n=10$ and 20 reveals that the impact of $\Delta n$ and $n$ on the performances due to the three sequential strategies can be different. Specifically, the predictive performances resulting from using IMSE and SAO seem more sensitive to $\Delta n$ and $n$ used, with IMSE performing slightly better with larger values of $\Delta n$ and $n$ while the converse holding true for SAO. The predictive performance due to BSAO is slightly better with larger values of $\Delta n$ and $n$ but it appears to be less sensitive to their values used. Table 4 shows the respective total numbers of design points added by the three design strategies. It is seen again that IMSE has the most design points added while SAO and BSAO have comparable numbers of design points used; also for all three design strategies, more design points are added when $\Delta n$ and $n$ are set at lower values.

\subsection{A Five-Dimensional Example}

The goal of this section is to compare the performances of SK delivered by using different sequential designs to predict $Y(\mathbf{x})$ over a five-dimensional design 


\begin{tabular}{|c|c|c|c|c|c|c|c|}
\hline \hline \multicolumn{3}{|c|}{$n=10, \Delta n=10, N=10^{3}$} & \multicolumn{4}{c|}{$n=20, \Delta n=20, N=10^{3}$} \\
\hline & IMSE & SAO & BSAO & & IMSE & SAO & BSAO \\
\hline min & 40 & 25 & 24 & min & 36 & 24 & 23 \\
\hline median & 53 & 34 & 33 & median & 43 & 29 & 28 \\
\hline mean & 53 & 33 & 33 & mean & 43 & 28 & 29 \\
\hline max & 67 & 43 & 46 & max & 48 & 34 & 35 \\
\hline
\end{tabular}

Table 4: A summary of the total numbers of design points used by different sequential design strategies for the 2D-Sine experiment

space $\mathscr{X}$, given a total budget of $N=2500$ simulation replications to expend. For the candidate design-point set, a two-layer nested Latin hypercube design (LHD) is used. The first layer $\mathbf{X}^{k_{0}}$ is a LHD with $k_{0}=50$ points and the full set $\mathbf{X}^{k_{\text {tot }}}$ is a LHD with $k_{\text {tot }}=250$ points, such that $\mathbf{X}^{k_{0}} \subset \mathbf{X}^{k_{\text {tot }}}$ is satisfied. The initial design is conveniently chosen as $\mathbf{X}^{k_{0}}$, a LHD of $k_{0}$ points itself, with $n=20$ simulation runs allocated at each point. Therefore, in each iteration, according to a given design strategy, we consider selecting points from $\mathbf{X}^{k_{\text {tot }}}$ to allocate $\Delta n=20$ simulation runs.

To evaluate predictive accuracy of SK, we construct the testing set $\mathbf{X}^{K}$ consisting of $K=2500$ Latin hyper cube sampled test points in $\mathscr{X}$. The entire experiment is repeated for 50 independent macro-replications, and the corresponding performance measure is set as ERMSE as defined by (12).

We examine a constructed example based on the borehole function (Morris et al., 1993) which has been widely used to illustrate various methods for deterministic computer experiments. The function models the flow rate of water through a borehole in $\mathrm{m}^{3} / \mathrm{yr}$, and Table 5 shows the eight variables of the function. For demonstration purposes, we use the following 5D-borehole function which is adapted from Equation (2.1) of (Morris et al., 1993) by fixing $H_{u}, H_{l}$ and $L$ at their respective lowest values:

$$
Y(\mathbf{x})=\frac{580 \pi x_{3}}{\ln \left(\frac{x_{2}}{x_{1}}\right)\left(1+\frac{2240 x_{3}}{\ln \left(\frac{x_{2}}{x_{1}}\right) x_{1}^{2} x_{5}}+\frac{x_{3}}{x_{4}}\right)},
$$

where $\mathbf{x}=\left(x_{1}, x_{2}, \ldots, x_{5}\right)^{\top}$, with the range of values for each $x_{i}$ as presented in Table 5. The simulated response at a point $\mathbf{x}$ on the $j$ th replication is given by $\mathscr{Y}_{j}(\mathbf{x})=\mathrm{Y}(\mathbf{x})+\varepsilon_{j}(\mathbf{x})$, where $\varepsilon_{j}(\mathbf{x})$ 's are i.i.d. $\mathscr{N}(0, \mathrm{~V}(\mathbf{x}))$ random variables with $\mathrm{V}(\mathbf{x})=0.1|\mathrm{Y}(\mathbf{x})|$. Table 6 provides a summary of the values of the true mean 


\begin{tabular}{ccccc}
\hline Variable & Denoted by & Description & Range & Unit \\
\hline$r_{w}$ & $x_{1}$ & radius of borehole & {$[0.05,0.15]$} & $\mathrm{m}$ \\
$r$ & $x_{2}$ & radius of influence & {$[100,50,000]$} & $\mathrm{m}$ \\
$T_{u}$ & $x_{3}$ & transmissivity of upper aquifer & {$[63,070,115,600]$} & $\mathrm{m}^{2} / \mathrm{yr}$ \\
$H_{u}$ & - & potentiometric head of upper aquifer & {$[990,1,110]$} & $\mathrm{m}$ \\
$T_{l}$ & $x_{4}$ & transmissivity of lower aquifer & {$[63.1,116]$} & $\mathrm{m}^{2} / \mathrm{yr}$ \\
$H_{l}$ & - & potentiometric head of lower aquifer & {$[700,820]$} & $\mathrm{m}$ \\
$L$ & - & length of borehole & {$[1,120,1,680]$} & $\mathrm{m}$ \\
$K_{w}$ & $x_{5}$ & hydraulic conductivity of borehole & {$[9,855,12,045]$} & $\mathrm{m}^{2} / \mathrm{yr}$ \\
\hline
\end{tabular}

Table 5: Parameters for the borehole problem

and variance functions at 2500 Latin hypercube sampled test points over 50 independent macro-replications. Despite using a 5D example here, we note that the proposed sequential design strategies can be adapted to address a problem with dimension $d>5$ so long as an adequate candidate design-point set can be constructed.

\begin{tabular}{|c|c|c|c|c|}
\hline \hline & max & mean & median & min \\
\hline $\mathrm{Y}(\mathbf{x})$ & 214.533 & 95.604 & 88.280 & 20.499 \\
& $(0.182)$ & $(0.009)$ & $(0.052)$ & $(0.033)$ \\
$\mathrm{V}(\mathbf{x})$ & 21.453 & 9.560 & 8.828 & 2.050 \\
& $(0.018)$ & $(8.581 \mathrm{E}-4)$ & $(0.005)$ & $(0.003)$ \\
\hline
\end{tabular}

Table 6: The mean and standard error (in parentheses) of the summary statistics for the values of the true mean and variance at a LHD of 2500 test points over 50 macro-replications

Results. Figure 4 summarizes the ERMSEs obtained using the three sequential design strategies with a total budget of $N=2500$ simulation replications. We observe that SAO and BSAO outperform IMSE by a relatively large margin with SAO and BSAO having comparable performances. Table 7 shows the respective total numbers of design points added by the three design strategies over 50 macroreplications. It is found that IMSE has the most design points added while SAO and BSAO have similar numbers of design points used; nevertheless, the numbers of design points added by the three strategies do not differ as much as observed in the $\mathrm{M} / \mathrm{M} / 1$ inspired example and the 2D-Sine example. A justification for this phenomenon is that, in contrast to the other two examples, the signal-to-noise ratio in this 5D-borehole example is much higher as manifested by a comparison of Table 6, Figures 1 (a)-(b), and Figure 3 (a)-(b); furthermore, with a higher 


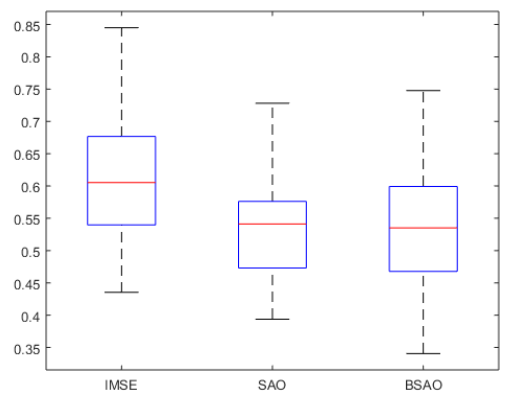

Figure 4: Boxplots of ERMSEs obtained from 50 macro-replications for the 5D-borehole experiment.

\begin{tabular}{|c|c|c|c|}
\hline \hline \multicolumn{4}{|c|}{$n=20, \Delta n=20, N=2500$} \\
\hline & IMSE & SAO & BSAO \\
\hline min & 84 & 72 & 74 \\
\hline median & 90 & 79 & 79 \\
\hline mean & 89 & 79 & 79 \\
\hline max & 97 & 84 & 83 \\
\hline
\end{tabular}

Table 7: A summary of the total numbers of design points used by different sequential design strategies for the 5D-borehole experiment

dimensional design space to explore it is more reasonable to spend the simulation budget on reducing the response-surface uncertainty instead of the sampling uncertainty. In a nutshell, we expect SAO and BSAO to strike a good balance between reducing the two sources of uncertainty in most problem contexts.

\section{Conclusions}

In this paper we have established some theoretical results on the predictive performance of SK and proposed three IMSE-based sequential design strategies to apply the SK metamodeling methodology for mean response prediction. The performance of SK with the proposed design strategies implemented is demonstrated through numerical examples of different features. Two sequential design strategies, BSAO and SAO, are found to be especially promising for helping SK achieve high predictive performance, as they account for the response-surface uncertainty and the sampling uncertainty more explicitly and adaptively. The use of these two strategies becomes more advantageous when the magnitude of the simulation variance is higher.

The paper only serves as a first step toward establishing efficient sequential design strategies to implement the SK methodology for stochastic simulation. It suggests several possible directions of future research. First, it is important to study the impact of the initial design and the step size on the predictive performance of SK applied with the sequential strategies proposed, with an emphasis on the roles that the step size $\Delta n$ and the initial number of simulation replications $n$ play. Second, a natural extension is to consider augmenting an initial design in a 
batch sequential manner, that is, to add several new design points to the existing design-point set on each iteration (see, e.g., Loeppky et al., 2010), which will facilitate exploiting the distributed computing power to enable a fast parallel update of SK metamodels (see Chevalier et al., 2015). Third, for high dimensional problems, it is imperative that we are able to construct an adequate candidate designpoint set and to update the set adaptively. Candidate design-point sets should encourage progressive exploration of the design space; and space-filling sequences such as Sobol sequences seem to be viable solutions. Fourth, for simulation models that each simulation run is prohibitively expensive, it will be helpful to study sequential design strategies that facilitate simultaneous construction of adequate heteroscedastic metamodels (see, e.g., Marrel et al., 2012; Binois et al., 2016) for both the mean and variance response surfaces so that the use of simulation replications at design points can be avoided. Fifth, we need to test the performance of the proposed sequential design strategies using more discrete-event simulation models. Last but not the least, we are interested in investigating sequential design strategies for global metamodeling to perform optimization in the stochastic simulation setting.

\section{Acknowledgments}

Earlier work that inspired this article were published in Chen and Zhou (2014). The authors thank the three referees for their constructive comments, which helped improve the manuscript significantly. The work of the first author is partially supported by the ICTAS Junior Faculty Award No. 176371 at Virginia Tech. 
A. Ajdari and H. Mahlooji. An adaptive exploration-exploitation algorithm for constructing metamodels in random simulation using a novel sequential experimental design. Communications in Statistics-Simulation and Computation, 43: 947-968, 2014.

B. E. Ankenman, B. L. Nelson, and J. Staum. Stochastic kriging for simulation metamodeling. Operations Research, 58:371-382, 2010.

J. Bect, D. Ginsbourger, L. Li, V. Picheny, and E. Vazquez. Sequential design of computer experiments for the estimation of a probability of failure. Statistics and Computing, 22:773-793, 2012.

J. M. Bekki, X. Chen, and D. Batur. Steady-state quantile parameter estimation: an empirical comparison of stochastic kriging and quantile regression. In A. Tolk, S. D. Diallo, I. O. Ryzhov, L. Yilmaz, S. Buckley, and J. A. Miller, editors, Proceedings of the 2014 Winter Simulation Conference, pages 3880-3891, Piscataway, New Jersey, 2014. Institute of Electrical and Electronics Engineers, Inc.

M. Binois, R. B. Gramacy, and M. Ludkovski. Practical heteroskedastic gaussian process modeling for large simulation experiments. 2016.

A. Boukouvalas and D. Cornford. Learning heteroscedastic gaussian processes for complex datasets. Technical report, Neural Computing Research Group, Aston University, 2009.

X. Chen and K.-K. Kim. Building metamodels for quantile-based measures using sectioning. In R. Pasupathy, S.-H. Kim, A. Tolk, R. Hill, and M. E. Kuhl, editors, Proceedings of the 2013 Winter Simulation Conference, pages 521-532, Piscataway, New Jersey, 2013. Institute of Electrical and Electronics Engineers, Inc.

X. Chen and K.-K. Kim. Stochastic kriging with biased sample estimates. $A C M$ Transactions on Modeling and Computer Simulation, 24:8/1-8/23, 2014.

X. Chen and K.-K. Kim. Efficient VaR and CVaR measurement via stochastic kriging. INFORMS Journal on Computing, 28:629-644, 2016.

X. Chen and Q. Zhou. Sequential experimental designs for stochastic kriging. In A. Tolk, S. Y. Diallo, I. O. Ryzhov, L. Yilmaz, S. Buckley, and J. A. Miller, editors, Proceedings of the 2014 Winter Simulation Conference, pages 3821-3832, 
Piscataway, New Jersey, 2014. Institute of Electrical and Electronics Engineers, Inc.

X. Chen, B. E. Ankenman, and B. L. Nelson. The effects of common random numbers on stochastic kriging metamodels. ACM Transactions on Modeling and Computer Simulation, 22:7/1-7/20, 2012.

X. Chen, B. E. Ankenman, and B. L. Nelson. Enhancing stochastic kriging metamodels with gradient estimators. Operations Research, 61:512-528, 2013.

C. Chevalier, X. Emery, and D. Ginsbourger. Fast update of conditional simulation ensembles. Mathematical Geosciences, 47:771-789, 2015.

K. T. Fang, D. K. J. Lin, P. Winker, and Y. Zhang. Uniform design: Theory and application. Technometrics, 42:237-248, 2000.

B. Gauthier and L. Pronzato. Spectral approximation of the IMSE criterion for optimal designs in kernel-based interpolation models. SIAM/ASA Journal on Uncertainty Quantification, 2:805-825, 2014.

B. Gauthier and L. Pronzato. Approximation of IMSE-optimal designs via quadrature rules and spectral decomposition. Communications in StatisticsSimulation and Computation, 45:1600-1612, 2016.

L. L. Gratiet and J. Garnier. Asymptotic analysis of the learning curve for Gaussian process regression. Journal of Machine Learning, 98:407-433, 2015.

Y. Hwang, X. He, and P. Z. G. Qian. Sliced orthogonal array-based Latin hypercube designs. Technometrics, 58:50-61, 2016.

D. R. Jones, M. Schonlau, and W. J. Welch. Efficient global optimization of expensive black-box functions. Journal of Global Optimization, 13:455-492, 1998.

B. Kamiński. A method for the updating of stochastic kriging metamodels. European Journal of Operational Research, 247:859-866, 2015.

K. Kersting, C. Plagemann, P. Pfaff, and W. Burgard. Most liekly heteroscedastic gaussian process regression. In Proceedings of the 24th International Conference on Machine Learning, ICML '07, pages 393-400. ACM, 2007. 
J. P. C. Kleijnen. Design and Analysis of Simulation Experiments. Springer, New York, 2nd edition, 2015.

J. P. C. Kleijnen. Regression and Kriging metamodels with their experimental designs in simulation: A review. European Journal of Operational Research, 256:1-16, 2017.

J. P. C. Kleijnen and W. C. M. van Beers. Application-driven sequential designs for simulation experiments: Kriging metamodeling. Journal of the Operational Research Society, 58:876-883, 2004.

A. M. Law. Simulation Modeling \& Analysis. McGraw-Hill, Inc, Boston, 5th edition, 2015.

M. Liu and J. Staum. Stochastic kriging for efficient nested simulation of expected shortfall. Journal of Risk, 12:3-27, 2010.

J. L. Loeppky, J. Sacks, and W. Welch. Choosing the sample size of a computer experiment: A practical guide. Technometrics, 51:366-376, 2009.

J. L. Loeppky, L. M. Moore, and B. J. Williams. Batch sequential designs for computer experiments. Journal of Statistical Planning and Inference, 140:1452-1464, 2010.

A. Marrel, B. Iooss, S. da Veiga, and M. Ribatet. Global sensitivity analysis of stochastic computer models with joint metamodels. Statistics and Computing, 22:833-847, 2012.

M. D. McKay, R. J. Beckman, and W. J. Conover. Comparison of three methods for selecting values of input variables in the analysis of output from a computer code. Technometrics, 21:239-245, 1979.

E. Mehdad and J. P. C. Kleijnen. Classic kriging versus kriging with bootstrapping or conditional simulation: classic kriging's robust confidence intervals and optimization. Journal of the Operational Research Society, 66:1804-1814, 2015.

M. D. Morris, T. J. Mitchell, and D. Ylvisaker. Bayesian design and analysis of computer experiments: Use of derivatives in surface prediction. Technometrics, 35:243-255, 1993. 
S. H. Ng and J. Yin. Bayesian kriging analysis and design for stochastic simulations. ACM Transactions on Modeling and Computer Simulation, 22:1-26, 2012.

C. Osorio and M. Bierlaire. A simulation-based optimization framework for urban transportation problems. Operations Research, 61:1333-1345, 2013.

L. Ouyang, Y. Ma, J. Wang, and Y. Tu. A new loss function for multi-response optimization with model parameter uncertainty and implementation errors. $E u$ ropean Journal of Operational Research, 258:552-563, 2017.

V. Picheny, D. Ginsbourger, Y. Richet, and G. Caplin. Quantile-based optimization of noisy computer experiments with tunable precision (with Comments and Rejoinder). Technometrics, 55:2-36, 2013.

P. Z. G. Qian. Nested latin hypercube designs. Biometrika, 96:957--970, 2009.

P. Ranjan, D. Bingham, and G. Michailidis. Sequential experiment design for contour estimation from complex computer codes. Technometrics, 50:527-541, 2008.

C. E. Rasmussen and C. K. I. Williams. Gaussian Processes for Machine Learning. MIT, Cambridge, 2006.

T. J. Robinson, J. B. Birch, and B. A. Starnes. A semi-parametric approach to dual modeling when no replication exists. Journal of Statistical Planning and Inference, 140:2860-2869, 2010.

T. J. Santner, B. J. Williams, and W. I. Notz. The Design and Analysis of Computer Experiments. Springer, New York, 2003.

M. I. Santos and P. M. Santos. Switching regression metamodels in stochastic simulation. European Journal of Operational Research, 251:142-147, 2016.

M. C. Shewry and H. P. Wynn. Maximum entropy sampling. Journal of Applied Statistics, 14:165-170, 1987.

B. Tang. Orthogonal array-based latin hypercubes. Journal of the American Statistical Association, 88:1392-1397, 1993. 
W. C. M. van Beers and J. P. C. Kleijnen. Customized sequential designs for random simulation experiments: Kriging metamodeling and bootstrapping. European Journal of Operational Research, 186:1099-1113, 2008.

W. Wang and X. Chen. The effects of estimation of heteroscedasticity on stochastic kriging. In T. M. K. Roeder, P. I. Frazier, R. Szechtman, E. Zhou, T. Huschka, and S. E. Chick, editors, Proceedings of the 2016 Winter Simulation Conference, pages 326-337, Piscataway, New Jersey, 2016. Institute of Electrical and Electronics Engineers, Inc.

W. Whitt. Planning queueing simulations. Management Science, 35:1341-1366, 1989.

F. Yang, B. E. Ankenman, and B. L. Nelson. Estimating cycle time percentile curves for manufacutring systems via simulation. INFORMS Journal on Computing, 20:628--643, 2008. 


\section{AppendixA. Proof of Theorem 1}

PROOF. Let $\Sigma_{\varepsilon}^{\Delta}=\operatorname{diag}\left\{\frac{\mathrm{V}\left(\mathbf{x}_{1}\right)}{n_{1}+\Delta n_{1}}, \frac{\mathrm{V}\left(\mathbf{x}_{2}\right)}{n_{2}+\Delta n_{2}}, \ldots, \frac{\mathrm{V}\left(\mathbf{x}_{k}\right)}{n_{k}+\Delta n_{k}}\right\}$, where $\mathrm{V}\left(\mathbf{x}_{i}\right)$ denotes the intrinsic variance at design point $\mathbf{x}_{i}$ and $n_{i}$ is the number of simulation replications already assigned there. Write $\Sigma_{\varepsilon}^{\Delta}=\Sigma_{\varepsilon}+\Delta \Sigma_{\varepsilon}$, where $\Delta \Sigma_{\varepsilon}=\operatorname{diag}\left\{C_{1}, C_{2}, \ldots, C_{k}\right\}$, and $C_{i}=\frac{\mathrm{V}\left(\mathbf{x}_{i}\right)}{n_{i}+\Delta n_{i}}-\frac{\mathrm{V}\left(\mathbf{x}_{i}\right)}{n_{i}}=\frac{-\mathrm{V}\left(\mathbf{x}_{i}\right) \Delta n_{i}}{\left(n_{i}+\Delta n_{i}\right) n_{i}}$ for $i=1,2, \ldots, k$. It is obvious that $C_{i} \leq 0$, $i=1,2, \ldots, k$, hence $\Delta \Sigma_{\varepsilon}$ is negative semi-definite.

Denote the $k \times k$ variance-covariance matrix after adding the $\Delta n$ replications by $\Sigma^{\Delta}:=\Sigma_{\mathrm{M}}+\Sigma_{\varepsilon}^{\Delta}$. Given an allocation of $\left\{\left(\mathbf{x}_{i}, n_{i}+\Delta n_{i}\right)\right\}_{i=1}^{k}$, the MSE of prediction at any prediction point $\mathbf{x}_{0}$ can be obtained by

$$
\operatorname{MSE}^{\Delta}\left(\mathbf{x}_{0}\right)=\tau^{2}-\tau^{4} \mathbf{r}_{0}^{\top}\left(\Sigma^{\Delta}\right)^{-1} \mathbf{r}_{0}
$$

where $\mathbf{r}_{0}=\left(r_{0,1}, r_{0,2}, \ldots, r_{0, k}\right)^{\top}$, where $r_{0, i}=\mathscr{R}\left(\mathbf{x}_{0}-\mathbf{x}_{i} ; \theta\right)$ for $i=0,1, \ldots, k$. Hence the IMSE follows as

$$
\begin{aligned}
\operatorname{IMSE}_{k}^{\Delta} & =\int_{\mathbf{x}_{0} \in \mathscr{X}} \operatorname{MSE}^{\Delta}\left(\mathbf{x}_{0}\right) d \mathbf{x}_{0} \\
& =\int_{\mathbf{x}_{0} \in \mathscr{X}} \tau^{2} d \mathbf{x}_{0}-\tau^{4} \mathbf{1}_{k}^{\top}\left[\mathbf{W}_{k} \circ\left(\Sigma^{\Delta}\right)^{-1}\right] \mathbf{1}_{k},
\end{aligned}
$$

where $\circ$ is the operator for Hadamard product. Assuming that $\Sigma=\Sigma_{M}+\Sigma_{\varepsilon}$ is invertible, applying the Woodbury identity gives

$$
\left(\Sigma^{\Delta}\right)^{-1}=\Sigma^{-1}-\Sigma^{-1}\left(\mathbf{I}_{k}+\Delta \Sigma_{\varepsilon} \Sigma^{-1}\right)^{-1} \Delta \Sigma_{\varepsilon} \Sigma^{-1}
$$

where $\mathbf{I}_{k}$ represents the $k \times k$ identity matrix. Substituting the equation above for $\left(\Sigma^{\Delta}\right)^{-1}$ into (A.1), we arrive at

$$
\begin{aligned}
\operatorname{IMSE}_{k}^{\Delta}= & \int_{\mathbf{x}_{0} \in \mathscr{X}} \tau^{2} d \mathbf{x}_{0}-\tau^{4} \mathbf{1}_{k}^{\top}\left[\mathbf{W}_{k} \circ \Sigma^{-1}\right] \mathbf{1}_{k} \\
& +\tau^{4} \mathbf{1}_{k}^{\top}\left[\mathbf{W}_{k} \circ\left(\Sigma^{-1}\left(\mathbf{I}_{k}+\Delta \Sigma_{\varepsilon} \Sigma^{-1}\right)^{-1} \Delta \Sigma_{\varepsilon} \Sigma^{-1}\right)\right] \mathbf{1}_{k} \\
= & \mathrm{IMSE}_{k}+\Delta \mathrm{IMSE}^{o}
\end{aligned}
$$

where IMSE $\mathrm{I}_{k}$ above denotes the IMSE obtained based on the allocation of $\left\{\left(\mathbf{x}_{i}, n_{i}\right)\right\}_{i=1}^{k}$, i.e., right before allocating the $\Delta n$ replications, and

$$
\Delta \mathrm{IMSE}^{o}=\tau^{4} \mathbf{1}_{k}^{\top}\left[\mathbf{W}_{k} \circ\left(\Sigma^{-1}\left(\mathbf{I}_{k}+\Delta \Sigma_{\varepsilon} \Sigma^{-1}\right)^{-1} \Delta \Sigma_{\varepsilon} \Sigma^{-1}\right)\right] \mathbf{1}_{k} .
$$


We note that $\Delta \mathrm{IMSE} \leq 0$ since $\Delta \Sigma_{\varepsilon}$ is negative semi-definite. Therefore, one wants to make $\Delta \mathrm{IMSE}^{o}$ as small as possible by optimally allocating the $\Delta n$ replications.

We next formulate a constrained optimization program to solve for the optimal budget allocation rule. Similar to Equations (26)-(28) given in Ankenman et al. (2010), we get

$$
\text { minimize } \Delta \operatorname{IMSE}(\Delta \mathbf{n})
$$

subject to

$$
\mathbf{1}_{k}^{\top} \Delta \mathbf{n} \leq \Delta n
$$

$\Delta n_{i}$ nonnegative integers,

where $\Delta \mathbf{n}=\left(\Delta n_{1}, \Delta n_{2}, \ldots, \Delta n_{k}\right)^{\top}$. Following the approach given by e-companion of Ankenman et al. (2010), we first formulate the Lagrangian as

$$
L(\Delta \mathbf{n})=\tau^{4} \mathbf{1}_{k}^{\top}\left[\mathbf{W}_{k} \circ\left(\Sigma^{-1}\left(\mathbf{I}_{k}+\Delta \Sigma_{\varepsilon} \Sigma^{-1}\right)^{-1} \Delta \Sigma_{\varepsilon} \Sigma^{-1}\right)\right] \mathbf{1}_{k}+\lambda\left(\Delta n-\mathbf{1}_{k}^{\top} \Delta \mathbf{n}\right) .
$$

The first-order optimality conditions are

$$
\begin{aligned}
\frac{\partial L(\Delta \mathbf{n})}{\partial \Delta n_{i}} & =\tau^{4} \mathbf{1}_{k}^{\top}\left[\mathbf{W}_{k} \circ \frac{\partial}{\partial \Delta n_{i}}\left(\Sigma^{-1}\left(\mathbf{I}_{k}+\Delta \Sigma_{\varepsilon} \Sigma^{-1}\right)^{-1} \Delta \Sigma_{\varepsilon} \Sigma^{-1}\right)\right] \mathbf{1}_{k}-\lambda \\
& =0, \quad i=1,2, \ldots, k .
\end{aligned}
$$

Now we have

$$
\frac{\partial}{\partial \Delta n_{i}}\left(\Sigma^{-1}\left(\mathbf{I}_{k}+\Delta \Sigma_{\varepsilon} \Sigma^{-1}\right)^{-1} \Delta \Sigma_{\varepsilon} \Sigma^{-1}\right)=\text { Part I }+ \text { Part II }
$$

where

$$
\begin{aligned}
\text { Part I } & =\frac{\partial}{\partial \Delta n_{i}}\left(\Sigma^{-1}\left(\mathbf{I}_{k}+\Delta \Sigma_{\varepsilon} \Sigma^{-1}\right)^{-1}\right) \Delta \Sigma_{\varepsilon} \Sigma^{-1} \\
& =-\Sigma^{-1}\left(\mathbf{I}_{k}+\Delta \Sigma_{\varepsilon} \Sigma^{-1}\right)^{-1} \frac{\partial}{\partial \Delta n_{i}}\left(\mathbf{I}_{k}+\Delta \Sigma_{\varepsilon} \Sigma^{-1}\right)^{-1}\left(\mathbf{I}_{k}+\Delta \Sigma_{\varepsilon} \Sigma^{-1}\right)^{-1} \Delta \Sigma_{\varepsilon} \Sigma^{-1} \\
& =\Sigma^{-1}\left(\mathbf{I}_{k}+\Delta \Sigma_{\varepsilon} \Sigma^{-1}\right)^{-1} \frac{\mathrm{V}\left(\mathbf{x}_{i}\right)}{\left(n_{i}+\Delta n_{i}\right)^{2}} \mathbf{J}^{(i i)} \Sigma^{-1}\left(\mathbf{I}_{k}+\Delta \Sigma_{\varepsilon} \Sigma^{-1}\right)^{-1} \Delta \Sigma_{\varepsilon} \Sigma^{-1}
\end{aligned}
$$

and

$$
\begin{aligned}
\text { Part II } & =\Sigma^{-1}\left(\mathbf{I}_{k}+\Delta \Sigma_{\varepsilon} \Sigma^{-1}\right)^{-1} \frac{\partial}{\partial \Delta n_{i}}\left(\Delta \Sigma_{\varepsilon}\right) \Sigma^{-1} \\
& =-\Sigma^{-1}\left(\mathbf{I}_{k}+\Delta \Sigma_{\varepsilon} \Sigma^{-1}\right)^{-1} \frac{\mathrm{V}\left(\mathbf{x}_{i}\right)}{\left(n_{i}+\Delta n_{i}\right)^{2}} \mathbf{J}^{(i i)} \Sigma^{-1}
\end{aligned}
$$


with $J^{(i i)}$ being the $k \times k$ matrix with one in the $i$ th diagonal position and zeroes elsewhere.

Now substituting Part I and Part II into (A.5), we arrive at for $i=1,2, \ldots, k$,

$$
\begin{aligned}
\frac{\partial L(\Delta \mathbf{n})}{\partial \Delta n_{i}}= & \tau^{4} \mathbf{1}_{k}^{\top}\left(\mathbf { W } _ { k } \circ \left[\Sigma^{-1}\left(\mathbf{I}_{k}+\Delta \Sigma_{\varepsilon} \Sigma^{-1}\right)^{-1} \mathbf{J}^{(i i)} \Sigma^{-1}\left(\mathbf{I}_{k}+\Delta \Sigma_{\varepsilon} \Sigma^{-1}\right)^{-1} \Delta \Sigma_{\varepsilon} \Sigma^{-1}\right.\right. \\
& \left.\left.-\Sigma^{-1}\left(\mathbf{I}_{k}+\Delta \Sigma_{\varepsilon} \Sigma^{-1}\right)^{-1} \mathbf{J}^{(i i)} \Sigma^{-1}\right]\right) \mathbf{1}_{k} \cdot \frac{\mathrm{V}\left(\mathbf{x}_{i}\right)}{\left(n_{i}+\Delta n_{i}\right)^{2}}-\lambda=0 .
\end{aligned}
$$

Now assume that $\max _{i=1, \ldots, k} \Delta \Sigma_{\varepsilon}(i, i) \ll 1$, such that $\mathbf{I}_{k}+\Delta \Sigma_{\varepsilon} \Sigma^{-1} \approx \mathbf{I}_{k}$. Then the equation above can be further simplified to the following form:

$$
\frac{\partial L(\Delta \mathbf{n})}{\partial \Delta n_{i}} \approx-\tau^{4} \mathbf{1}_{k}^{\top}\left(\mathbf{W}_{k} \circ\left[\Sigma^{-1} \mathbf{J}^{(i i)} \Sigma^{-1}\right]\right) \mathbf{1}_{k} \cdot \frac{\mathrm{V}\left(\mathbf{x}_{i}\right)}{\left(n_{i}+\Delta n_{i}\right)^{2}}-\lambda=0, i=1,2, \ldots, k .
$$

By denoting $Q_{i}:=\tau^{4} \mathbf{1}_{k}^{\top}\left(\mathbf{W}_{k} \circ\left[\Sigma^{-1} \mathbf{J}^{(i i)} \Sigma^{-1}\right]\right) \mathbf{1}_{k}$ for $i=1,2, \ldots, k$, similar arguments as those given by Ankenman et al. (2010) give that

$$
n_{i}+\Delta n_{i}^{*} \propto \sqrt{Q_{i} \mathrm{~V}\left(\mathbf{x}_{i}\right)}, i=1,2, \ldots, k
$$

\section{AppendixB. Proof of Theorem 2}

PROOF. In the same spirit to (A.1), we obtain the following expression for the IMSE,

$$
\begin{aligned}
\operatorname{IMSE}_{k+1} & =\int_{\mathscr{X}} \operatorname{MSE}_{k+1}\left(\mathbf{x}_{0}\right) d \mathbf{x}_{0} \\
& =\int_{\mathscr{X}} \tau^{2} d \mathbf{x}_{0}-\tau^{4} \mathbf{1}_{k+1}^{\top}\left[\mathbf{W}_{k+1} \circ\left(\Sigma_{k+1}\right)^{-1}\right] \mathbf{1}_{k+1},
\end{aligned}
$$

where $k+1$ in the subscript denotes the size of the design-point set upon adding a new design point $\mathbf{x}_{k+1}$.

On the other hand, we have

$$
\Sigma_{k+1}=\Sigma_{\mathrm{M}, k+1}+\Sigma_{\varepsilon, k+1}=\left(\begin{array}{cc}
\tau^{2}+\frac{\mathrm{V}\left(\mathbf{x}_{k+1}\right)}{\Delta n} & \tau^{2} \mathbf{r}_{k+1,}^{\top} \\
\tau^{2} \mathbf{r}_{k+1, \cdot} & \Sigma_{k}
\end{array}\right)
$$

Assuming that $\Sigma_{k}$ is invertible, we apply the blockwise inversion formula and obtain

$$
\Sigma_{k+1}^{-1}=\ell\left(\begin{array}{cc}
1 & -\tau^{2} \mathbf{r}_{k+1}^{\top}, \Sigma_{k}^{-1} \\
-\tau^{2} \Sigma_{k}^{-1} \mathbf{r}_{k+1,} . & \tau^{4} \Sigma_{k}^{-1} \mathbf{r}_{k+1,}, \mathbf{r}_{k+1,}^{\top}{ }^{-1}+\Sigma_{k}^{-1} \ell^{-1}
\end{array}\right)
$$


where $\ell=\left(\operatorname{MSE}_{k}\left(\mathrm{Y}\left(\mathbf{x}_{k+1}\right)\right)+\frac{\mathrm{V}\left(\mathbf{x}_{k+1}\right)}{\Delta n}\right)^{-1}$ is a scalar, and $\operatorname{MSE}_{k}\left(\mathrm{Y}\left(\mathbf{x}_{k+1}\right)\right)$ is simply the MSE obtained in predicting at the $(k+1)$ th design point by using the existing set of $k$ design points.

Now by plugging (8) and (B.3) into (B.1), through some algebraic manipulation we arrive at the following expression for $\mathrm{IMSE}_{k+1}$,

$$
\begin{aligned}
& \mathrm{IMSE}_{k+1}=\int_{\mathscr{X}} \operatorname{MSE}_{k+1}\left(\mathbf{x}_{0}\right) d \mathbf{x}_{0} \\
& =\int_{\mathscr{X}} \tau^{2} d \mathbf{x}_{0}-\tau^{4} \mathbf{1}_{k}^{\top}\left[\mathbf{W}_{k} \circ\left(\Sigma_{k}\right)^{-1}\right] \mathbf{1}_{k} \\
& -\tau^{4} \ell\left(w_{k+1, k+1}-2 \tau^{2} \mathbf{1}_{k}^{\top}\left[\mathbf{w}_{k+1, \cdot} \circ \Sigma_{k}^{-1} \mathbf{r}_{k+1,}\right]\right. \\
& \left.+\mathbf{1}_{k}^{\top}\left[\mathbf{W}_{k} \circ\left(\Sigma_{k}^{-1} \mathbf{r}_{k+1,}, \mathbf{r}_{k+1,}^{\top}, \Sigma_{k}^{-1}\right)\right] \mathbf{1}_{k}\right), \\
& =\operatorname{IMSE}_{k}+\Delta \mathrm{IMSE}\left(\mathbf{x}_{k+1} ; \Delta n\right),
\end{aligned}
$$

and

$$
\Delta \operatorname{IMSE}\left(\mathbf{x}_{k+1} ; \Delta n\right)=-\tau^{4} \ell \mathbf{1}_{k+1}^{\top}\left[\mathbf{W}_{k+1} \circ \mathbf{E}\right] \mathbf{1}_{k+1} .
$$

It is not hard to see that $\Delta \operatorname{IMSE}\left(\mathbf{x}_{k+1}\right) \leq 0$ and hence the IMSE decreases. 